\title{
Zippering and Intermeshing: Novel Phase Diagrams for Interfaces and Films
}

\author{
Peter B. Weichman and Anoop Prasad \\ Condensed Matter Physics 114-36, California Institute of Technology, Pasadena, California 91125
}

(Received 10 July 1995)

\begin{abstract}
New surface and layering phase diagrams are proposed based on generalized sine-Gordon models with and without a substrate potential. In particular, we find that the preroughening transition can be driven first order, explaining "zipper" features in heat capacity data for argon and krypton on graphite substrates. For different parameters, we predict the existence of a novel variant of den Nijs' disordered flat phase with spontaneously broken particle-hole symmetry and continuously varying surface height with an accompanying intermeshing layering phase diagram. The restricted solid-on-solid model displays zippering for sufficiently large second neighbor coupling.
\end{abstract}

PACS numbers: 64.60.-i, 68.35.Rh, 68.55.Jk, 82.65.Dp

Roughening and preroughening phenomena on bulk crystal-vapor interfaces occur when entropic forces that encourage a more disordered surface overcome energetic forces that prefer a flat surface. Roughening occurs via a Kosterlitz-Thouless transition [1] to a phase with logarithmically diverging mean square surface height difference,

$$
\begin{aligned}
G\left(\mathbf{r}-\mathbf{r}^{\prime}\right) & \equiv \frac{1}{2}\left\langle\left[h(\mathbf{r})-h\left(\mathbf{r}^{\prime}\right)\right]^{2}\right\rangle \\
& \approx\left[4 \pi K_{R}(T)\right]^{-1} \ln \left(\left|\mathbf{r}-\mathbf{r}^{\prime}\right| / a_{0}\right),
\end{aligned}
$$

for large $\left|\mathbf{r}-\mathbf{r}^{\prime}\right|$. Here $h(\mathbf{r})$ is the integer height over a 2D lattice labeled by $\mathbf{r}, K_{R}(T)$ is the fully renormalized tilt modulus, and $a_{0}$ is of order the lattice spacing. Preroughening is more subtle [2,3] and involves a transition to a disordered flat (DOF) phase in which the surface is macroscopically flat $[G(r \rightarrow \infty)$ is finite], but microscopically rough. The top layer has fractional occupancy $\theta$, which at preroughening jumps from $\theta_{F}=$ 0 to $\theta_{\mathrm{DOF}}=\frac{1}{2}$. The transition occurs when thermal fluctuations overcome the local (e.g., nearest neighbor) interactions, but stronger, longer ranged (e.g., second neighbor) interactions exist to stabilize an overall flat interface [2]. At higher $T$ the interface finally roughens completely.

Recent experiments involving the growth of thin films on a substrate $[4,5]$ have been interpreted as strong evidence for preroughening behavior [6]. The key feature is a sequence of reentrant first order layering transitions between integer-plus-one-half coverages lying above the usual low temperature layering transitions between integer coverages (see Fig. 1). These are suggested to be the thin film analog of the DOF phase. However, an unexplained feature is a zigzagging line of transitions forming a "zipper" between the two (see Fig. 2 and Fig. 1 of [6]). The first purpose of this Letter is to show that, under certain conditions, the preroughening transition can become first order, leading to the zipper. The second is to show that under different conditions den Nijs' $\theta$ DOF phase [3], with continuously varying upper layer coverage, $0 \leq \theta(T) \leq \frac{1}{2}$, can appear via a new mechanism involving spontaneous breaking of particlehole symmetry. In thin films this leads to an as yet unobserved phenomenon of intermeshing of the two sets of layering lines (see Fig. 3).

Roughening and preroughening transitions are dominated by universal long wavelength critical fluctuations. In renormalization group language, the behavior is controlled by a fixed point (actually, a fixed line in this case). This allows a complete description of the large lengthscale physics using a coarse grained Hamiltonian which depends only on a few effective parameters. For roughening phenomena the fixed point region may be charac-

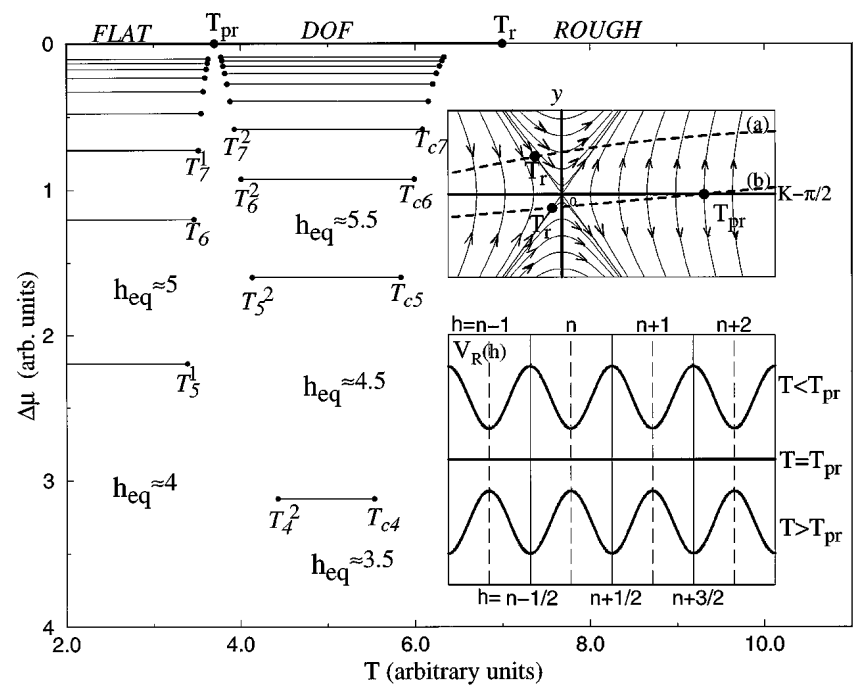

FIG. 1. Preroughening and reentrant layering. Solid lines indicate first order transitions between integer $\left(T<T_{\mathrm{pr}}\right)$ or halfinteger $\left(T>T_{\mathrm{pr}}\right)$ film thicknesses (the first few lie off scale). The phase diagram for the bulk interface, $\Delta \mu=0$, is shown along the top. The upper inset shows examples of physical trajectories, $\left[K_{0}(T), y_{0}(T)\right]$, overlayed on renormalization group flows for $\kappa=u \equiv 0$ : (a) roughening; (b) preroughening followed by roughening, as detailed in the main part of the figure, where we have used $K_{R}\left(T_{\mathrm{pr}}\right)-\pi / 2=1$. The lower inset shows the evolution of the renormalized corrugation potential, $V_{R}(h ; T)$, with $T$ along trajectory (b) near $T_{\mathrm{pr}}$. 


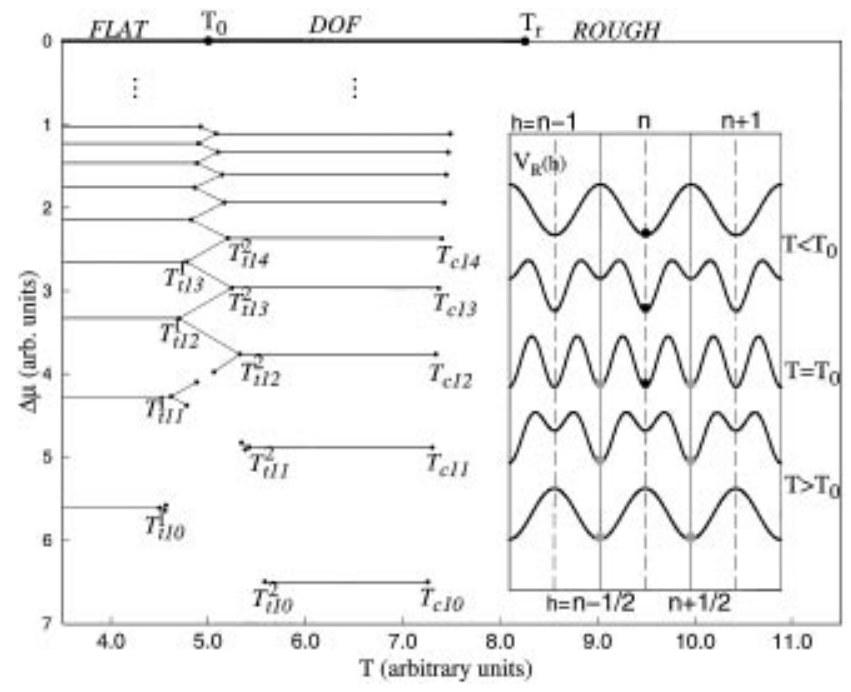

FIG. 2. First order preroughening and zippering $\left(u_{R}>0\right)$. We take $u_{0}(T) \equiv 10^{-5}$ and $K_{0}\left(T_{0}\right)=12$. The unzipping phenomenon is evident in the thinner films. The inset shows the evolution of the renormalized corrugation potential with $T$. The stable minima jump from integer to half integer at $T_{0}$.

terized simply and completely by a sine-Gordon type reduced Hamiltonian, $\overline{\mathcal{H}}_{\mathrm{SG}} \equiv \mathcal{H}_{\mathrm{SG}} / k_{B} T[1]$ :

$$
\begin{aligned}
\overline{\mathcal{H}}_{\mathrm{SG}} & =\int d^{2} r\left\{\frac{1}{2} K_{0}|\nabla h(\mathbf{r})|^{2}+V_{0}[h(r)]\right\}, \\
V_{0}[h] & =-y_{0} \cos (2 \pi h)-u_{0} \cos (4 \pi h)+V_{\text {sub }}[h],
\end{aligned}
$$

where $h(\mathbf{r})$ now represents a coarse grained continuous surface height field, $K_{0}(T)$ is the renormalized tilt modulus, $y_{0}(T)$ represents the fundamental Fourier component of the renormalized atomic corrugation poten-

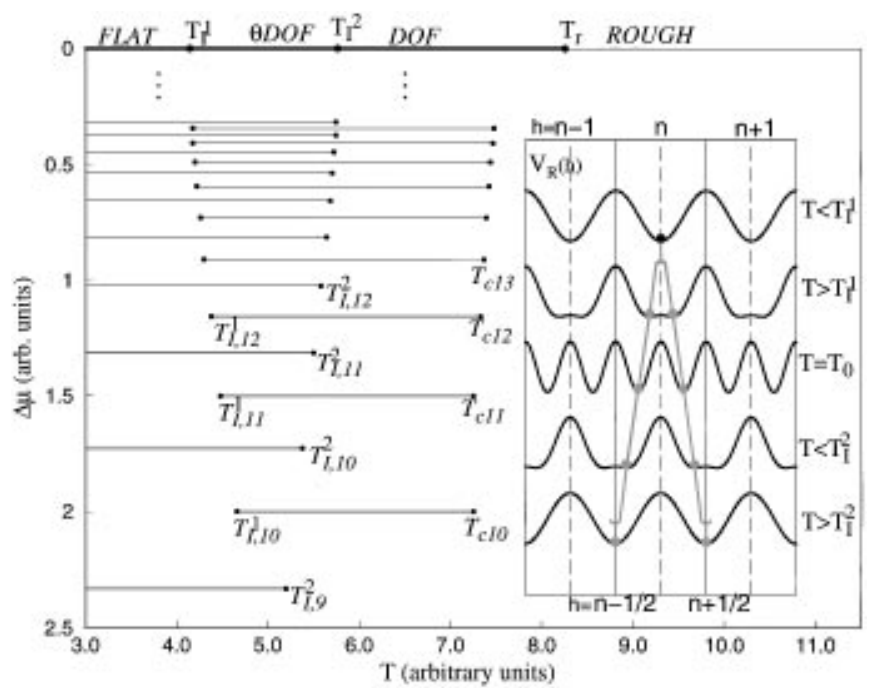

FIG. 3. The $\theta$ DOF phase and intermeshing $\left(u_{R}<0\right)$. We use $u_{0}(T) \equiv-10^{-5}$ and $K_{0}\left(T_{0}\right)=12$. The inset shows the evolution of the corrugation potential with $T$. The stable minima now evolve continuously from integer to half integer in the interval $T_{I}^{1}<T<T_{I}^{2}$. tial, $u_{0}(T)$ the next harmonic, and $V_{\text {sub }}[h]$ is the effective substrate potential. In order to discuss zippering and intermeshing we must keep $u_{0} \neq 0$. All higher harmonics, however, are strongly irrelevant [1] and may be assumed to vanish in our effective theory. The substrate potential serves mainly to define an average film thickness, $h_{0}(\Delta \mu) \sim \Delta \mu^{-1 /(1+\alpha)}$, with $\alpha=2$ for a van der Waals attraction, diverging as the deviation $\Delta \mu=\mu_{0}(T)-\mu$ of the chemical potential from that of the bulk interface vanishes [5]. The form (2) is valid only in the thick film limit where $V_{\text {sub }}[h]$ is extremely weak. The quadratic confining potential $V_{\text {sub }}[h] \approx \frac{1}{2} \kappa_{0}\left(h-h_{0}\right)^{2},\left[h-h_{0}\right] \ll h_{0}$, with a renormalized curvature $\kappa_{0}(T, \Delta \mu) \sim \Delta \mu^{(2+\alpha) /(1+\alpha)}$, suffices for thick films. Huse [7] has written down general functional recursion relations for any sufficiently weak potential $V_{0}[h]$, and treated in detail the interplay between roughening and layering.

To formalize what we have said so far we write down the renormalization group recursion relations for the Hamiltonian (2):

$$
\begin{aligned}
\dot{K}(l) & =\kappa^{2} / 2 K \Lambda^{4}+\left(4 \pi^{4} / K \Lambda^{4}\right) y^{2}+\left(64 \pi^{4} / K \Lambda^{4}\right) u^{2}, \\
\dot{y}(l) & =(2-\pi / K) y+\left(4 \pi^{2} / K \Lambda^{2}\right) y u, \\
\dot{u}(l) & =(2-4 \pi / K) u-\left(\pi^{2} / K \Lambda^{2}\right) y^{2}, \\
\dot{\kappa}(l) & =2 \kappa-\kappa^{2} / K \Lambda^{2},
\end{aligned}
$$

where $\Lambda \sim \pi / \alpha$ is the (nonuniversal) momentum space cutoff due to the lattice. The flow parameter $l$ is related to the spatial rescaling factor $b$ via $b=e^{l} ; u=\kappa \equiv 0$ yield the familiar Kosterlitz flow equations [1]. The recursion relations are valid for small $y, u$, and $\kappa$ and we have the initial conditions $K(l=0)=K_{0}(T)$, etc.

The macroscopic thermodynamics is determined by the large $l$ behavior of the flow trajectory for a given initial condition. Thus, imagine integrating the flow equations either until a fixed point is reached or until the trajectory exits the region of validity of Eq. (3). In the latter case, we stop integrating on some noncritical matching surface bounding this region at some value $l=l^{*}\left(K_{0}, y_{0}, u_{0}, \kappa_{0}\right)$ of the flow parameter. In either case, the final result is a Hamiltonian of the same form as (2), but with renormalized parameters $K_{R}(T)$ and $\quad V_{R}[h] \equiv-y_{R}(T) \cos (2 \pi h)-u_{R}(T) \cos (4 \pi h)+$ $\frac{1}{2} \kappa_{R}(T)\left(h-h_{0}\right)^{2}$. Whenever $V_{R}[h]$ is nonzero the interface will be confined near its minimum. In our computations we shall, for simplicity, ignore the small residual fluctuations and take the equilibrium interface height $h_{\text {eq }}$ to be precisely at this minimum.

It is easy to confirm that $y=u=\kappa=0$, but $K$ arbitrary, is a fixed line. Since any small $\kappa_{0}$ grows exponentially, the fixed line is reached only for a bulk interface. The fixed line is stable to $y$ only if $K<\pi / 2$ and to $u$ only if $K<2 \pi$. For thick films $\kappa(l)$ is always very small and can be neglected in the first three recursion relations, to that $y_{R}, u_{R}$, and $K_{R}$ take their $\kappa=0$ values, while $\kappa_{R} \approx \kappa_{0} \exp \left(2 l^{*}\right)$. 
Let us now consider the various possible behaviors. Consider first the substrate free case $\kappa_{0}=0$. For $K_{0} \lesssim$ $\pi / 2, y, u \rightarrow 0$ as $l \rightarrow \infty$, while the stiffness $K(l) \rightarrow$ $K_{R}(T)$, its fully renormalized value. The result is a purely Gaussian Hamiltonian and $G(\mathbf{r})$ then takes the form (1). In the presence of a substrate, the renormalized Hamiltonian is still Gaussian, but acquires a "mass" $\kappa_{R}$. This cuts off the growth of $G(\mathbf{r})$ and confines the interface to a region of width proportional to $\ln (1 / \Delta \mu)$ about $h_{0}$. Since $V_{R}[h]$ is smooth and featureless the film will grow continuously with $h_{0}$.

For intermediate values $\pi / 2 \lesssim K_{0} \lesssim 2 \pi, u$ is still irrelevant, and may be ignored, but if $y_{0} \neq 0, y(l)$ eventually begins to grow (see the upper inset to Fig. 1), as does $K(l)$, and we stop integrating at some fixed small $y=y_{R}$ (we take $y_{R}=0.6$ ). The strengthening corrugation potential and increasing tilt modulus signal the onset of a flat phase. Notice that if $y_{0}>0$ the minima occur at integer $h$, while if $y_{0}<0$ they occur at half integer $h$. If we now include $\kappa_{R}$, the degeneracy is broken, and for the given $h_{0}$ a unique $h_{\mathrm{eq}}$ is usually selected. Only for special values of $h_{0}$ do two neighboring minima become degenerate. As $h_{0}$ passes through one of these degeneracy points $h_{\text {eq }}$ jumps discontinuously from one minimum to the other, signifying a first order phase transition. For $y_{0}>0$ this transition is between approximately integer film thicknesses, while for $y_{0}<0$ it is between approximately half-integer film thicknesses. As $h_{0}$ increases an infinite sequence of first order transitions occurs, signifying layer by layer film growth. As $K_{0}$ decreases toward the roughening transition, these layering lines terminate in Ising critical points $T_{c, n}$, beyond which the continuous film growth detailed above occurs. The $T_{c, n}$, converge to the bulk roughening temperature $T_{r}$ from below as $T_{r}-T_{c, n} \sim 1 / \ln ^{2}(n)$ as $n \rightarrow \infty$ [7].

To understand preroughening, suppose that the effective parameter $y_{0}(T)$ changes sign, with $y_{0}\left(T_{\mathrm{pr}}\right)=0$. If this happens for $\pi / 2 \lesssim K_{0}\left(T_{\mathrm{pr}}\right) \lesssim 2 \pi$, the minima in $V_{R}[h]$ then switch abruptly from integer to half integer, consistent with the physics of preroughening at $T=T_{\mathrm{pr}}$ [3]. The sign reversal is driven precisely by the entropy of small scale roughness discussed above. For $y_{0}=0$ the fixed line is again stable, and we will have $u(l) \rightarrow 0$ and $K(l) \rightarrow$ $K_{R}\left(T_{\mathrm{pr}}\right)$, with a nonuniversal $\pi / 2<K_{R}\left(T_{\mathrm{pr}}\right)<2 \pi$. The critical interface is therefore rough, but with a larger renormalized stiffness than is generically possible.

On the preroughening line, the addition of the substrate potential leads to continuous film growth identical to that in the rough regime. Below $T_{\mathrm{pr}}$ the integer layering lines end in Ising critical points $T_{n}^{1}$, while above $T_{\mathrm{pr}}$ the reentrant half-integer layering lines begin and end in Ising critical points $T_{n}^{2}$ and $T_{c, n}$. Following [7], one finds that $T_{\mathrm{pr}}-$ $T_{n}^{1}, T_{n}^{2}-T_{\mathrm{pr}} \sim n^{-p\left(T_{\mathrm{pr}}\right)}$ with a nonuniversal exponent $p\left(T_{\mathrm{pr}}\right)=\frac{1}{2}(2+\alpha)\left[2-\pi / K_{R}\left(T_{\mathrm{pr}}\right)\right]$, while $T_{r}-T_{c, n}$ behave precisely as before. This behavior is shown in Fig. 1, and in Fig. 3 of [6].
Finally, for even larger $K_{0}\left(K_{0} \gtrsim 2 \pi\right)$ both $y$ and $u$ are relevant, so even if $y_{0}=0, u$ will grow to localize the interface. If $y_{0}$ is not precisely zero then both $u$ and $y$ will grow under renormalization and both $u_{R}$ and $y_{R}$ will be nonzero. We shall see that interference between the two has very strong effects on the phase diagrams.

We continue to assume that $y_{0}\left(T_{0}\right)=0$ for some temperature $T_{0}$. For numerical convenience we choose $l^{*}$ so that $\max \left\{10^{3}\left|u\left(l^{*}\right)\right|, 2\left|y\left(l^{*}\right)\right|\right\}=1$. The flow equations then imply that $y_{R}(T) \propto T-T_{0}$ for $T$ near $T_{0}$. Suppose first that $u_{R}\left(T_{0}\right)>0$. The evolution of the corrugation potential with $T$ is then shown in the inset to Fig. 2. We see that at no point does the corrugation potential vanish, and the interface is therefore always flat. As $y_{R}(T)$ decreases, new minima develop at $h=n+\frac{1}{2}$, and precisely at $T_{0}$ they become degenerate with those at $h=n$. For $T>T_{0}, h=n+\frac{1}{2}$ are the absolute minima and there is a first order phase transition from a flat to a DOF phase. The layering phase diagram is even more interesting. When $y_{R}$ is of the same order as $\kappa_{R}$ and $h_{0}=n+\frac{1}{2}$, the curvature due to the substrate potential is sufficient to make $h=n+\frac{1}{2}$ the absolute minimum even when $y_{R}>0$. This means that there is an interval in temperature around $T_{0}$ when integer and half-integer coverages are alternately stable as $h_{0}$ increases. One finds that the integer layering lines end in triple points $T_{t, n}^{1}$, out of which emerge two new first order lines which then join up to the two neighboring triple points $T_{t, n}^{2}$ that begin the reentrant half-integer layering lines. This is the promised zippering behavior (Fig. 2). It can be shown that $T_{0}-T_{t, n}^{1}, T_{t, n}^{2}-T_{0} \sim n^{-q}$ as $n \rightarrow \infty$ with $q=2+\alpha$ (which simply reflects the fact that $\kappa_{R} \sim$ $\kappa_{0} \sim n^{-q}$ scale identically in this first order regime). As $K_{0}$ decreases toward the preroughening regime the behavior converts to Fig. 1 by unzipping the zipper from the bottom. Thus, as $\kappa_{R} / u_{R}$ increases the zigzagging transitions break in the middle (bottom part of Fig. 2), and retract into the triple point, at which precise point, $y_{R}=16 u_{R}=\kappa_{R} / 3 \pi^{2}$, it is tricritical and then, for larger $\kappa_{R} / u_{R}$, critical.

Now suppose that $u_{R}\left(T_{0}\right)<0$. The evolution of the corrugation potential is shown in the inset to Fig. 3. At a temperature $T_{1}^{I}$, such that $y_{R}=4 u_{R}$, the minima split continuously into two via an Ising phase transition, signifying a continuously varying surface height $n \pm$ $\theta(T)$. Just above $T_{I}^{1}$ one has $\theta(T) \sim\left(T-T_{I}^{1}\right)^{1 / 8}$, and at $T_{0}$ one has $\theta\left(T_{0}\right)=\frac{1}{4}$. Above $T_{0}$ the minima at $n+\theta$ and $n+1-\theta$ move together until at a temperature $T_{I}^{2}$, such that $y_{R}=-4 u_{R}$, they merge in a second Ising critical point and $\theta(T)=\frac{1}{2}$ for $T \geq T_{I}^{2}$. Just below $T_{I}^{2}$ one will have then $\frac{1}{2}-\theta(T) \sim\left(T_{I}^{2}-T\right)^{1 / 8}$. The intermediate phase $T_{I}^{1}<T<T_{I}^{2}$ is called the $\theta \mathrm{DOF}$ phase. Such a phase is generated, even when $u=0$, by particle-hole symmetry breaking corner interactions, which generate a term $-y_{0}^{\prime} \sin (2 \pi h)$ in $V_{0}[h]$ [3]. Here this phase is generated spontaneously by the interference 


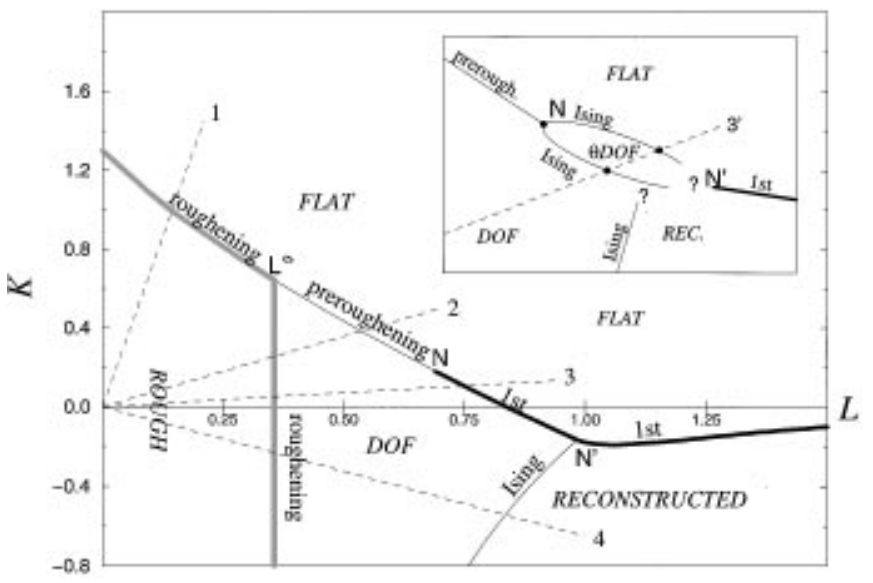

FIG. 4. Global surface phase diagram, along with various possible experimental trajectories (with 2, 3, and $3^{\prime}$ treated in the first three figures), for the RSOS model on a square lattice computed using a four spin plaquette mean field theory [6]. Strong evidence for $u_{R}>0$ is observed. The mean field theory does not actually produce true roughening behavior, so the corresponding (thick grey) lines are determined partially empirically and partially taken from [3]. All other features, however, are exhibited directly by the theory. The inset shows schematically the behavior expected in the neighborhood of $N$ and $N^{\prime}$ if $u_{R}<0$, including the new $\theta$ DOF phase. Since we have not yet found a model with $u_{R}<0$, we do not fully understand how things connect up. Reconstruction, and hence the Ising line, are absent for a triangular lattice substrate [6], vastly increasing the size of the DOF phase and the range of first order preroughening, but leaving the remainder of the phase diagram qualitatively unaffected.

between $u$ and $y$ when $K$ is sufficiently large. As $K_{0}$ decreases toward the preroughening region, the two Ising lines come closer together, merging to become the continuous preroughening line precisely when $u$ becomes irrelevant (inset to Fig. 4).

In the presence of $\kappa_{R}$, for $T_{I}^{1} \lesssim T \lesssim T_{I}^{2}$, there will again be twice as many first order layering lines. However, the fact that all minima are now exactly degenerate, the transitions always occur when $h_{0}$ is either integer or half integer. These lines must then end in Ising critical points $T_{I, n}^{1}$ and $T_{I, n}^{2}$, with $T_{I, n}^{1}-T_{I}^{1}, T_{I}^{2}-T_{I, n}^{2} \sim n^{-q}$. This is the intermeshing behavior shown in Fig. 3.

Which of the four possible behaviors we have discussed is exhibited by a particular model or system depends on the detailed interactions. If $y_{0}$ does not change sign, then only a single set of layering transitions will be observed. If it does change sign, and $K_{0}\left(T_{0}\right)$ is sufficiently large, either preroughening behavior will be seen or, depending on the sign of $u_{R}$, zippering or intermeshing will be seen. The experimental systems of argon and krypton on graphite [5] clearly show behavior consistent with Fig. 2 . We have investigated via a plaquette mean field theory $[6,8]$ the restricted solid-on-solid (RSOS) model [2,3] of the interface for both square and triangular (appropriate to experiment) lattices. Parameters are nearest neighbor coupling $K=$ $J_{1} / k_{B} T$, and second neighbor coupling $L=J_{2} / k_{B} T$. The resulting global phase diagram (including reconstruction, which we have not addressed in this Letter), along with various possible experimental trajectories, is shown in Fig. 4, and should be contrasted with Fig. 1 in [3]. There is clear evidence for an interval (between the points $N$ and $N^{\prime}$ in the figure) of first order preroughening, and hence $u>0$, before the surface reconstructs. We should caution, however, that the mean field theory may overestimate the distance between $N$ and $N^{\prime}$, and that the first order nature of the transition is rather weak, and this may be why the two points were indistinguishable in earlier studies $[2,3]$. Note, however, that reconstruction and hence $N^{\prime}$ are absent on the triangular lattice, so the validity of our theory, and the associated existence of zippering, is essentially beyond doubt in this case.

We have yet to find a RSOS type model with $u_{R}<0$. However, the Ashkin-Teller model has the same sineGordon representation and shows a preroughening line that forks into two Ising lines, precisely consistent with $u<0$ [9]. How to translate this observation into an appropriate interface model is still under investigation. We also plan simulations of realistic atomic systems to see how the effective parameters depend upon the interatomic potentials and, with luck, to guide searches for appropriate new experimental systems.

This research was supported by the Sloan Foundation and the NSF under Grant No. DMR-9308205. We thank David Goodstein for highly productive interactions.

[1] See, e.g., J. V. Jose, L.P. Kadanoff, S. Kirkpatrick, and D. R. Nelson, Phys. Rev. B 16, 1217 (1977), and references therein.

[2] K. Rommelse and M. den Nijs, , Phys. Rev. Lett. 59, 2578 (1987); M. den Nijs and K. Rommelse, Phys. Rev. B 40, 4709 (1989).

[3] M. den Nijs, Phys. Rev. Lett. 64, 435 (1990); M. den Nijs, Phase Transitions in Surface Films, edited by H. Taub et al. (Plenum, New York, 1992), Vol. 2.

[4] H. S. Youn and G. B. Hess, Phys. Rev. Lett. 64, 918 (1990); H. S. Youn, X.F. Meng, and G. B. Hess, Phys. Rev. B 48, 14556 (1993).

[5] P. Day, M. Lysek, M. LaMadrid, and D. Goodstein, Phys. Rev. B 47, 10716 (1993); P. Day, M. LaMadrid, M. Lysek, and D. Goodstein, Phys. Rev. B 47, 7501 (1993).

[6] P. B. Weichman, P. Day, and D. Goodstein, Phys. Rev. Lett. 74, 418 (1995).

[7] D. Huse, Phys. Rev. B 30, 1371 (1984).

[8] P. B. Weichman, A. Prasad, and P. Day (to be published).

[9] M. Kohmoto, M. den Nijs, and L. P. Kadanoff, Phys. Rev. B 24, 5229 (1981). 